\title{
A Short Review of Architecture and Computational Analysis in the Design of Graphene-based Bioelectronic Devices
}

\author{
Amgad Ahmed Ali Ibrahim, ${ }^{1}$ Nik Noor Nabilah Md Ibrahim, ${ }^{1}$ \\ Anthony Centeno, ${ }^{2}$ and Abdul Manaf Hashim ${ }^{1 *}$ \\ ${ }^{1}$ Malaysia-Japan International Institute of Technology, Universiti Teknologi Malaysia, \\ Jalan Sultan Yahya Petra, 54100 Kuala Lumpur, Malaysia \\ ${ }^{2}$ Department of Electrical and Electronic Engineering, Xi'an Jiaotong-Liverpool University, \\ 111 Ren'ai Road, Suzhou Dushu Lake Higher Education Town, Jiangsu Province, 215123 China
}

(Received November 14, 2017; accepted March 19, 2018)

Keywords: graphene, biosensors, finite element analysis, density functional theory, computational analysis

Graphene possesses a high surface-to-volume ratio, which enables biomolecules to attach to it for bioelectronic applications. In this article, first, the classification and applications of bioelectronic devices are briefly reviewed. Then, recent work on real fabricated graphenebased bioelectronic devices as well as the analysis of their architecture and design using a computational approach to their charge transport properties are presented and discussed. A comparison to nongraphitic bioelectronic devices is also given. On the macroscale level, the design of devices is elaborated on the basis of a finite element analysis (FEA) approach, and the impact of design on the performance of the devices is discussed. On the nanoscale level, transport phenomena and their mechanisms for different design categories are elaborated on the basis of the density functional theory (DFT) and other quantum chemistry calculations. The calculated and measured charge transport properties of graphene-based bioelectronic devices are also compared with those of other available bioelectronic devices.

\section{Introduction}

Bioelectronic devices are defined as electronic devices that function by interacting with biomolecules such as human or animal tissues for the purpose of detection, actuation, or even power generation. Generally, bioelectronic devices may be classified into several categories on the basis of their applications as summarized in Fig. 1. As illustrated in Fig. 1, bioelectronic devices can be used for power generation in the form of enzymatic and microbial biofuel cells, ${ }^{(1-3)}$ and for testing and diagnostic purposes in the form of e-skin devices, nucleic acid amplification chips, DNA extraction chips, and glucose monitoring devices. ${ }^{(4-6)}$ For applications in artificial perception, several types of bioelectronic devices such as the bioelectronic nose, brain, and tongue have been demonstrated. ${ }^{(7,8)}$ In the past decade, on-chip bioelectronic devices with the unique function of mimicking human organs such as the kidney, *Corresponding author: e-mail: abdmanaf@utm.my http://dx.doi.org/10.18494/SAM.2018.1805 


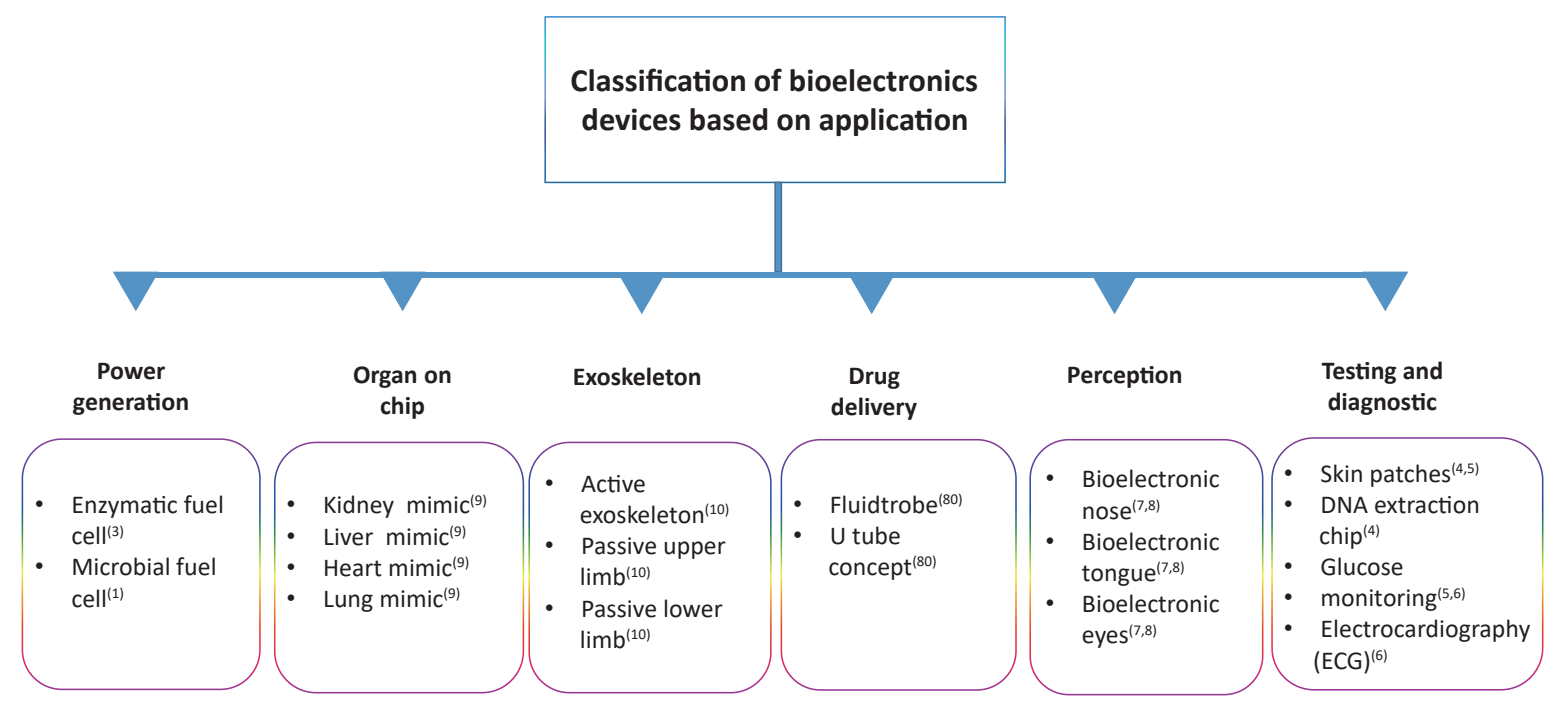

Fig. 1. (Color online) Classification of bioelectronic devices based on their applications.

lung, heart, and liver have been developed for use in medical treatment. ${ }^{(9)}$ Bioelectronic devices have also found their way into the development of exoskeletons for which a passive exoskeleton and active limbs have been designed and fabricated. ${ }^{(10)}$ Furthermore, bioelectronic devices are also reported for use in drug delivery. ${ }^{(2,11,12)}$ Electrochemical sensors or biosensors, which are also one type of bioelectronic device, have been the subject of both basic and applied research for nearly fifty years. ${ }^{(13-15)}$ Clark et al. first introduced the principle of the enzyme electrode with an immobilized glucose oxidase at the New York Academy of Sciences Symposium in 1962.(16) Currently, there are many examples of device commercialization based on such biosensing principles including those for pathogens and toxins. ${ }^{(17-19)}$ For example, an enzyme in the biorecognition layer acting as an electroactive substance was utilized in a multichannel electrochemical biosensor. ${ }^{(6,15,20)}$ Here, detection occurs owing to the physicochemical transduction that provides a measurable signal. Generally, a native enzyme is used as a biorecognition component, i.e., as an analyte ${ }^{(2,12,22)}$ or an inhibitor. ${ }^{(12,22)}$ In addition, enzymes can also be used as labels bonded to antibodies, antigens, or oligonucleotides with a specific sequence, thus providing affinity-based sensors. ${ }^{(15,21,23,24)}$

A similar principle has been extended to power generation, where an enzymatic biofuel cell has been developed in response to industrial demand. Such a bioelectronic device can generate energy in a sustainable manner by utilizing biocatalysts, either in the form of isolated enzymes ${ }^{(1,2)}$ or in the presence of enzymes within microbial cells ${ }^{(3)}$ to convert chemical energy into electricity. ${ }^{(1,3,25)}$ After a breakthrough study on the preparation of graphene, which is a single-atom-thick sheet of carbon, by Novoselov and co-workers, ${ }^{(26)}$ there has been tremendous effort to utilize this nanomaterial for the electronic coupling of redox enzymes. ${ }^{(27-30)}$ Its ultrahigh in-plane electron conductivity, ${ }^{(31,32)}$ high thermal conductivity, ${ }^{(33)}$ and mechanical properties $^{(31-33)}$ make it a promising material not only for the channel of electronic devices ${ }^{(34-36)}$ but also for the construction of bioelectrodes and biomembranes. ${ }^{(37-39)}$ Figure 2 shows a graphene-based field-effect transistor (FET) biosensor in which anti-immunoglobulin G 


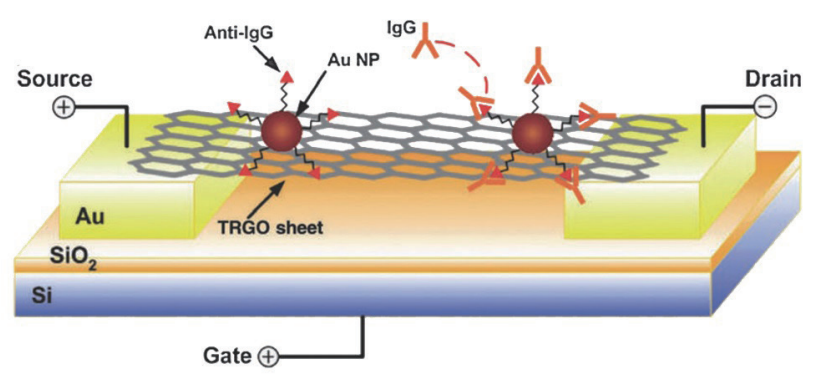

Fig. 2. (Color online) Graphene-based FET biosensor. ${ }^{(40)}$

(Anti-IgG) is anchored on gold nanoparticles (AuNPs). ${ }^{(40-42)}$ The AuNPs are spread over a thermally reduced graphene oxide (TrGO) membrane as the channel material of the FET; these nanoparticles have shown specific recognition ability for IgG. ${ }^{(41-43)}$ Such graphene-FET biosensors have been shown to exhibit exceptional sensitivity with detection limits down to 0.01 nM. Aptamer-modified graphene-FET biosensors also show label-free, real-time responses against immunoglobulin E (IgE) protein at very low concentrations. ${ }^{(42,44)}$ This sensor enables the electrical detection between aptamer and protein at low concentrations by optimizing the Debye length (DL), i.e., the distance required for screening the surplus charge. A bioelectronic nose based on a multiplexed graphene-FET system prepared by the micropatterning of graphene using photolithography was also developed for a human sensory mimicking system that can discern a specific odorant in a mixture..$^{(7,23,45)}$ Figure 3 shows the design and architecture of a multiplexed bionose fabricated using microstructured graphene-based FETs and the corresponding human olfactory system. The bionose was designed to detect odorants such as amyl butyrate and helional. In this structure, the gate of the FET is controlled by an aqueous ionic solution. Large-area-grown graphene with high conductance and large surface area combined with human olfactory will give high selectivity towards odorant mixtures. ${ }^{(45)}$ The olfactory sensory neurons are located at the entrance of the nasal cavity, where each of these neurons is separately coupled to a cluster of nerve endings at the olfactory bulb. As shown in Fig. 3, the olfactory neurons that are linked to certain olfactory receptors are attached to the same nerve ending. The interaction between odorants and their precise human olfactory receptors generates olfactory signals that are transferred through a cluster of neurons to olfactory bulbs. These signals are then processed by the human brain to recognize odorants by their smell. Correspondingly, the multiplexed bionose is designed and developed to mimic each step of the human olfactory system whereas the FET function is used to generate olfactory signals as well as an imitated olfactory code that combines numerous signals from a cluster of receptors. Using a template that resembles the transmembrane structure of the G-protein-coupled receptor and a structural model of the human olfactory receptor leads to the development of an excellent structure for an artificial bionose that can mimic the human nose. ${ }^{(7,23,45)}$ The use of graphene in those FET biosensors as an electrochemical transducer shows an exceptionally highly sensitive response owing to the extreme sensitivity of graphene 


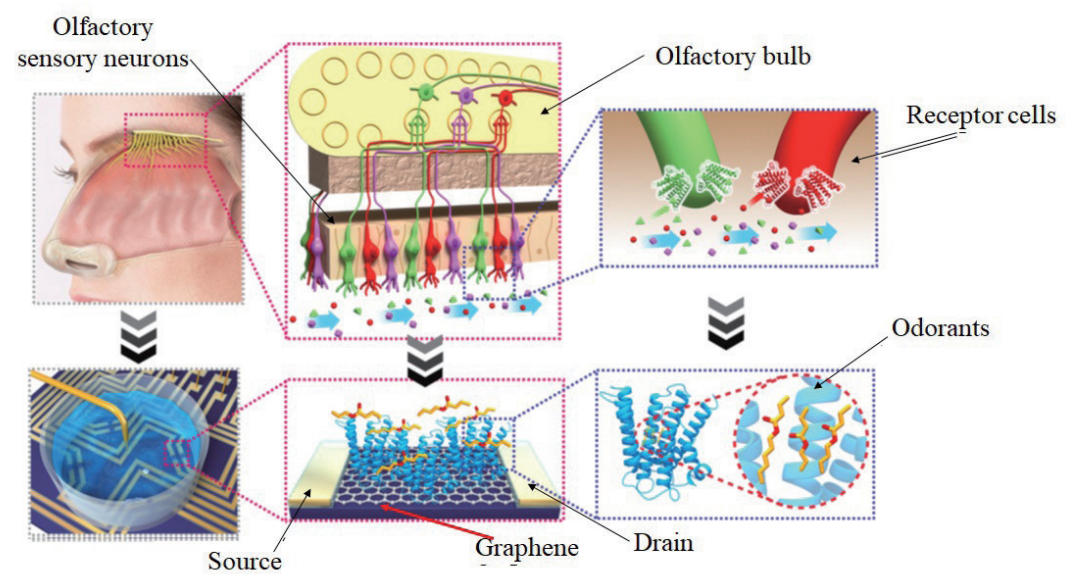

Fig. 3. (Color online) Design and architecture of a bionose. ${ }^{(7,23,45)}$

to electronic perturbations and its surrounding environment. ${ }^{(6)}$ Graphene with one atom thickness and high surface area allows a selective detection at single-carbon resolution, which leads to the development of highly sensitive FET biosensors. ${ }^{(7)}$

Another growing area in biodevices is the microfluidic-based organs-on-chip (OOC) systems with advanced 3D tissue engineered scaffolds combined with cultured human cells to replicate a human organ of interest. ${ }^{(9,45,46)}$ Microfluidic channel networks are designed and fabricated to mimic the organ structure, e.g., liver sinusoid or nephron in a kidney. The channel surfaces are usually modified with layers mimicking the extracellular matrix, allowing human cells to adhere, spread, and proliferate within the channels, thus requiring tissue engineering technologies. Once OOCs are constructed, a fluid flow is applied to generate mechanical forces that recapitulate the in vivo microenvironment experienced by cells. ${ }^{(46-48)}$ Specifically, organspecific fluid flow enables gradient formations of molecular components and maintenance of cell-cell interactions, ${ }^{(49,50)}$ which are vital to emulating human physiological responses. A pressure sensor is an important component in a microfluidic system in order to control and monitor fluid flow precisely. Precise pressure of the fluid is needed in order to stimulate the cultured cells. The use of graphene in a pressure sensor connected to a microfluidic system will allow for the sensitive and accurate measurement of the fluid pressure owing to its exceptional thermal and mechanical properties. A graphene pressure sensor monitors the changes in electrical resistance, and this will be the indication of detection regarding cell morphologies, contractile functions, and gene expression. ${ }^{(51)}$ Figure 4(a) shows a schematic representation of the structure of a kidney-on-chip device. ${ }^{(46,47)}$ A porous polymeric biocompatible membrane such as polydimethylsiloxane (PDMS) is sandwiched between epithelium and endothelium tissues. The former tissue lines the cavities, surfaces of blood vessels, and organs through the body. The structure of this tissue, as shown in Fig. 4(a), is in the form of a continous film with almost no intercellular space, where the unit cell is in a cuboidal form in most cases with nuclei at the center. ${ }^{(46,47)}$ On the other hand, the structure of endothelium tissue is similar to that of epithilium tissue, but the endothilial cells are elongated and aligned in the direction of fluid flow. Such unique structures impose a restriction on the transport of any migrating 


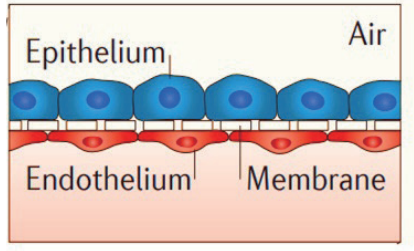

(a)

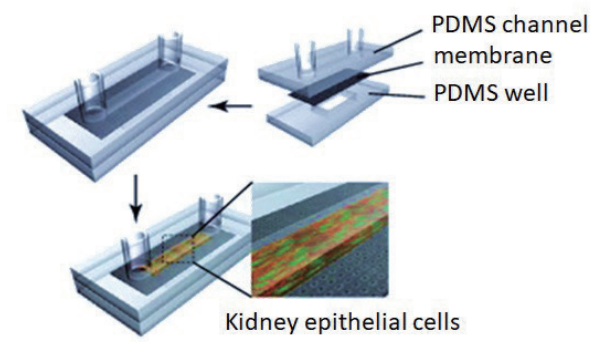

(b)

Fig. 4. (Color online) (a) Schematic representation of the structure of a kidney-on-chip device and (b) a free-body diagram equivalent to OOCs for computational purposes. ${ }^{(45,47)}$

species through or across the hybrid structure. ${ }^{(46,47)}$ A free-body diagram that is equivelant to the OOCs for computational purposes is shown in Fig. 4(b). The equivalent model of the microfluidic kidney epithelium is based on a multilayered microstructure that includes the arranged layers of PDMS microchannels and a PDMS chamber. The chamber is separated from the microchannels by a porous membrane made of polyester. This architecture offers a transport medium that is physically equivalent to polarized kidney epithelial cells. Hence, it accurately mimics the mechanism of fluidic flows and maintains the selective contact of apical and basal sides of the cells to fluid shear, hormones, and chemical gradients. In addition, this architecture enables the collection of samples from both sides of the polarized tissue. ${ }^{(46,47)}$

Similar to a regular fuel cell, a biofuel cell includes both an anode and a cathode, where any part of it must be a bioelectrode (an electrode that involves biocatalysts). For instance, in the case of the anode as the bioelectrode, the supplied fuel is oxidized at the bioelectrode by the biocatalyst, where a group of electrons are transferred (donated) from the biocatalyst to the bioanode. The biocatalyst also enables the reduction of groups in contact with oxygen at the surface of the biocathode as depicted in Fig. 5(a). ${ }^{(52)}$ Thus, to maintain high power output from the biofuel cell, it is important to preserve high catalytic activity at the bioelectrode surface. This may be achieved by selecting a proper biocatalyst and by embedding it in the bioelectrode, taking into consideration the optimum orientation once immobilized. The optimum orientation is achieved by aligning the catalyst along the conductive interface of an electrode in a way that enables direct electron transfer (DET). In order to achieve DET, the use of nanomaterials, i.e., graphene is needed to allow for the increase in electrocatalysis reaction. Graphene as a conductive agent will allow the further increase in the surface area of the electrode without changing its geometric dimensions, and with its unique properties, graphene can be used in a biofuel cell to provide good anchoring sites for catalyst deposition, and thus improve the performance of the biofuel cell. ${ }^{(52)}$ With the effective graphene-biocatalyst reaction, it can provide the networked electrode interfaces, and thus increase the biocatalyst loading. In this manner, the biocatalyst effectively allows the exchange of electrons within the electrode, and thus increase the biofuel cell output. Recently, a promising novel concept has been developed to maintain high catalytic bioactivity, and a versatile family of new conductive nanomaterials was synthesized. In this concept, 3D graphitic structures were decorated with catalytic metals 
(a)

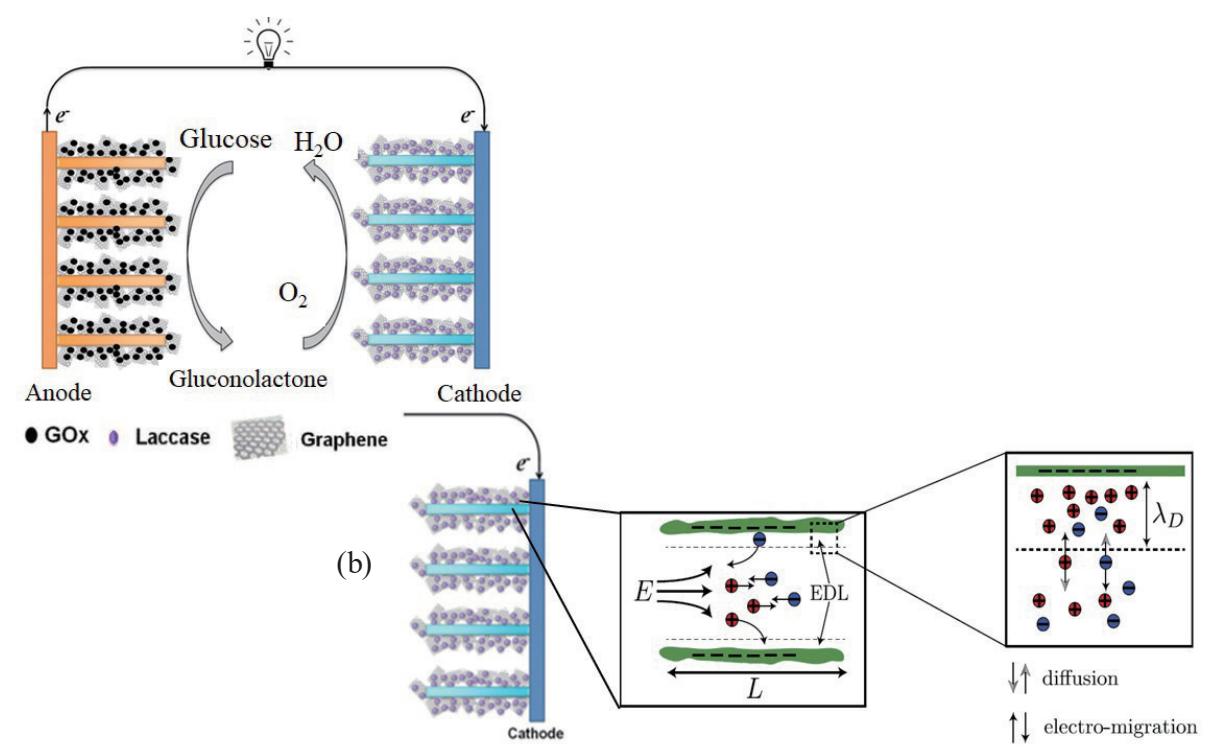

Fig. 5. (Color online) (a) Schematic representation of biofuel cell and (b) transport mode through composite electrode. ${ }^{(52,53)}$

such as $\mathrm{Pt}, \mathrm{Ni}$, or Pd. The composite nature of the electrodes results in different transport modes for charged particles similar to the transport in porous electrodes shown in Fig. 5(b). ${ }^{(53)}$ Upon the introduction of a potential difference between the electrodes, an electric field $(E)$ is generated to force the ions into the pores of the electrodes. The ions of opposite charge are attracted to the electrode, while the ions with the same charge are forced away from the surface. This successfully rearranges the charges at the electrodes, resulting in the formation of a shallow interface layer known as the electric double layer (EDL) with dimensions of length $L$ and radius $\lambda_{d}$. A remarkable property of the EDL is that it behaves as a capacitor under steadystate conditions. Thus, the flow of charges across the pores of the electrodes proceeds until the diffusion and electromigration of the ions reach equilibrium. ${ }^{(53)}$ These modes of transport must be carefully addressed during the modelling of the transport dynamics through such electrodes.

\section{Macroscale Analysis of Bioelectronic Devices}

Most bioelectronic devices are electrochemical. However, optical, calorimetric, piezoelectric, and surface plasmon resonance-based bioelectronic devices are also common. The schematic diagram presented in Fig. 6 shows the basic architecture of most bioelectronic devices. ${ }^{(54)}$ A bioelectronic device has a substrate that acts as a receptor for the specimens that contain the species of interest, such as DNA, dopamine, or glucose. The main role of this substrate is to attract, absorb, and transport the species of interest to the next stage of the device (the membrane) in addition to supporting the mechanical structure of the device in most scenarios. The next stage of the bio-electrochemical process is the filtration and separation of species of interest from their carrier and transporting them to the immobilized detector. Such 


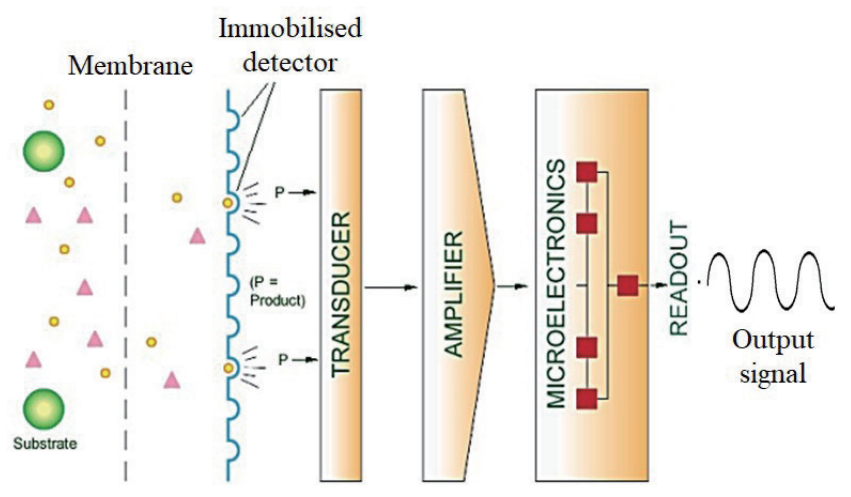

Fig. 6. (Color online) Schematic diagram of the basic components of a bioelectronic device. ${ }^{(54)}$

a step is usually carried out by functionalizing the membrane. The mechanical structure and the chemical composition of the membrane are critical design parameters for any bioelectronic device. In fact, these two parameters are the determinants of the device type. For instance, having a nanoporous bare graphene layer as a membrane could result in the ability to sense a single molecule of DNA in an ionic solution. On the other hand, using poly(vinyl alcohol) hydrogel as a membrane might result in an enzymatic biofuel cell. One of the most important modules that must be taken into consideration during the design of a bioelectronic device is the immobilized detector. The detector is a module responsible for detecting the separated species of interest (after it has been transported through the membrane) and enhancing its flow towards the transducer. A well-tailored immobilized detector results in a low-noise signal at the transducer, which is a feature of merit for any bioelectronic device. The electronic signal is collected from the transducer and amplified and may be further processed using microelectronics to capture the response.

The rate-determining step in several biological routines is electrodiffusion. For instance, the intertransmission of electrochemical signals within neurons through synapses is an electrodiffusion-dependant process. Another example is the electrodiffusion-controlled reaction that enables ligand-enzyme complexion. ${ }^{(55)}$ In fact, in these biological processes, the rates and orders of the reactions are mainly dependent on progressive electrodiffusion of charged particles or complexes through the transport medium. Particle-based computational models such as Langevin and Brownian dynamics (LD and MD) and the Monte Carlo (MC) method are widely used to predict reaction rates and constants for biological processes. ${ }^{(56,57)}$ Actually, particlebased computational methods depend mainly on the modelling of the asymptotic trajectory of a single particle in a specific energy frame in a discrete stochastic manner. Accordingly, simulations based on such models are difficult to converge in the case of multiparticle systems when the number of particles is large. To overcome this drawback of particle-based models, another computational model, namely, a continuum model, is used to compute reaction rates and constants. This model takes into consideration the distribution of charged particles through a system of differential equations that define the average density distribution. In this way, the 
continuum models are more capable than the particle-based models to deal with multiparticle large systems. ${ }^{(53,58,59)}$ In addition, such models may be coupled with established dynamic models such as Nernst-Planck, Nernst-Einstein, and Navier-Stokes models to integrate various modes of physical-chemical phenomena. These capabilities led the continuum electrodiffusion models to be more favoured for modelling the interaction of ion channels in biological processes ${ }^{(53,58,59)}$ and for describing ionic transport in ceramic and polymeric membranes as well as modelling the transport of charged particles in semiconductors. ${ }^{(53,58,59)}$

Figure 7 depicts a schematic of an ion channel bioprotonic device ${ }^{(60)}$ that could be considered for the general case of bioelectronic device architecture. A supported lipid bilayer (SLB) (orange spheres with tails) is sandwiched in an electrolytic layer (a polymeric membrane, graphene membrane, or a liquid electrolyte). The continuum of the cell includes different types of channels and pores. Various ionic species travel across the cell and the membrane from both sides. Two electrodes are the source of ohmic contacts, where the current density flows across it to the whole circuitry. This representation could be a schematic for an OOC device, a bioprotonic device, or in the case in which the SLB is replaced with a bulk material, it could be a schematic for a biosensor, or even a biofuel cell. In the next section, we discuss the transport of charged species across each part of this architecture.

Mass transfer through electrochemical bioelectronic devices can be considered to be governed by the Nernst-Planck equation that characterizes the fluxes of mobile solutes. The molar flux of species, $\phi_{i}$, is described as ${ }^{(55)}$

$$
\phi_{i}(x)=-D_{i} \frac{\partial a_{i}(x)}{\partial x}-\frac{z_{i} F}{R T} D_{i} C_{i} \frac{\partial \varphi(x)}{\partial x}+C_{i} v(x)
$$

where $D_{i}, a_{i}, C_{i}, z_{i}, v_{i}$, and $r_{k}$ indicate the diffusivity $\left(\mathrm{m}^{2} / \mathrm{s}\right)$, chemical activity, concentration $(\mathrm{mM})$, valence number, stoichiometric constants of the surface reaction, and reaction rate for the $i$ th mobile species with respect to the boundaries, respectively. The term $R$ is the universal gas constant $(8.314 \mathrm{~J} / \mathrm{mol} \mathrm{K}), T$ is the absolute temperature $(\mathrm{K}), F$ is the Faraday constant $(96487$ $\mathrm{C} / \mathrm{mol}$ ), and $\varphi$ is the electrochemical potential of the transport medium.

The Nernst-Planck equation [Eq. (1)] is formulated for a highly reactive ionic species, where

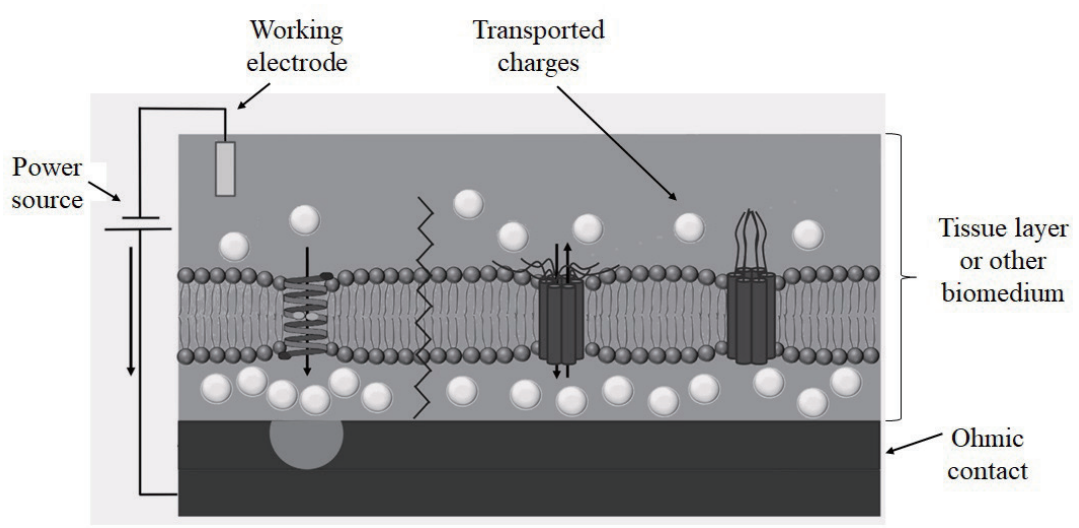

Fig. 7. Schematic representation of common bioelectronic device architecture. ${ }^{(60)}$ 
the chemical potential gradient is the driving force for transport as well as the electrostatic potential,

$$
\begin{aligned}
\frac{\partial a_{i}(x)}{\partial x} & \rightarrow \frac{\partial a_{c i}}{\partial x}, \\
\frac{\partial \phi}{\partial x} & \rightarrow \frac{\partial \psi}{\partial x},
\end{aligned}
$$

where $\psi$ is the electrostatic potential. In this case, Eq. (1) could be written as

$$
\phi_{i}(x)=-\nabla \cdot\left[-D_{i} \nabla c_{i}+\vec{\mu}_{i} c_{i}\right],
$$

where $\vec{\mu}_{i}$ is the velocity field vector that is related to the electrostatic potential as $\vec{\mu}_{i}=\vec{\mu}-\mu_{E P} \nabla \psi$. Here, $\vec{\mu}$ is the fluid velocity vector and $\mu_{E P} \nabla \psi$ is the electrophoretic velocity of ions.

However, for the bioelectronic applications, the terms of activity and electrochemical potential may be changed. For a nonionic solute where the charge $z_{k}=0$, the Nernst-Planck Eq. (1) is reduced to Fick's first law of diffusion. The motion of the ionic species ensures the current transport through the electrolyte(s) in which each ionic species carries a current density, $J_{i}$, proportional to its charge as given by Eq. (4).

$$
J_{i}=z_{i} F \phi_{i}
$$

Equation (1) can be written as

$$
\phi_{i}=\phi_{\text {diff }, i}+\phi_{m i g, i}+\phi_{c o n v, i},
$$

where $\phi_{\text {diff }, i}$ is the diffusive transport, $\phi_{m i g, i}$ is the migration driven by electrostatic potential, and $\phi_{\text {mig, } i}$ is the convective transport.

Actually, the diffusive transport $\phi_{\text {diff }, i}$ may be neglected in the case of outflow. In such situations, either convective or migration or both transport modes control the transport process; thus, the Nernst-Planck flux equation may be written as

$$
n \cdot(-D \nabla C)=0,
$$

where $n$ is the unit vector perpendicular to the outflow flux boundaries. In this sense, the transport of species through the membrane maintains a planner flux even though the concentration changes in three-dimensional space. This practice should save computation time and yet maintain accuracy.

\section{Bulk Membrane Transport}

Bulk membrane transport is a concept that describes the movement of a group of charges across (perpendicular to a membrane's axis of symmetry) a membrane in bulk. In such 
transport, charged species do not cross the membrane in a single ion queue but instead they are either clustered or aggregated in a cloud. Another reason for the nomenclature of this transport type is that the moving species need to cross a bulk film such as composite graphene membranes, a poreless membrane, or a plasma membrane. Figure 8 shows a schematic representation of transport of different ionic species through graphene oxide (GO) bulk membrane transport. In this type of transport medium, ions are allowed to permeate through the GO membrane. The permeability of ions is size dependent; for instance, $\mathrm{Mg}^{2+}$ (radius $0.72 \AA$ ) cations have higher permeability than $\mathrm{Fe}^{3+}$ (radius 0.6 $\AA$ ). Furthermore, it was reported that, in addition to the size effect, the interactions between the transporting ions and the GO layers are mainly controlled by the selectivity of the GO membranes. In fact, the $\pi$-bonding-based interactions between the GO atoms and the transported alkaline cations are weaker than the coordinative interactions between transition metal ions and the $\mathrm{sp}^{3}$ matrix of the GO layers. However, the coordination of a soft metal cation $\mathrm{Cd}^{2+}$ seems to be weaker than those of $\mathrm{Cu}^{2+}$ and $\mathrm{Fe}^{3+}$, possibly due to the larger distance between $\mathrm{Cd}^{2+}$ and the oxygen-containing functional groups in the matrix. In addition, it appears that the concentration of a cation could also be affected by its source. For instance, the concentrations of $\mathrm{Cu}^{2+}$ and $\mathrm{Cd}^{2+}$ originating from $\mathrm{CuCl}_{2}$ and $\mathrm{CdCl}_{2}$ were both higher than those from $\mathrm{CuSO}_{4}$ and $\mathrm{CdSO}_{4}$. This finding indicates that the through-membrane transport of a cation could be controlled by the electrostatic attraction of its counteranion. ${ }^{(61,62)}$

In a bioelectronic device, abrupt differential changes in the activity of species might take place at the electrode and separator surfaces as shown in Fig. 8. In the bulk medium, the activity gradients are not remarkable, and the current is driven mostly by migration rather than convection. Thus, using the migration term from Eq. (4), the current density of transported ionic species through the bulk medium can be formulated as

$$
J_{i}=J_{m i g, i}=-\lambda_{i} C_{i} \frac{\partial \varphi(x)}{\partial x} .
$$

Here, $\lambda_{i}$ denotes the molar ionic conductivity $\left(\mathrm{m}^{2} \mathrm{~S} / \mathrm{mol}\right)$ and is related to the transport parameters by

$$
\lambda_{i}=D_{i} \frac{z_{i}^{2} F^{2}}{R T}
$$

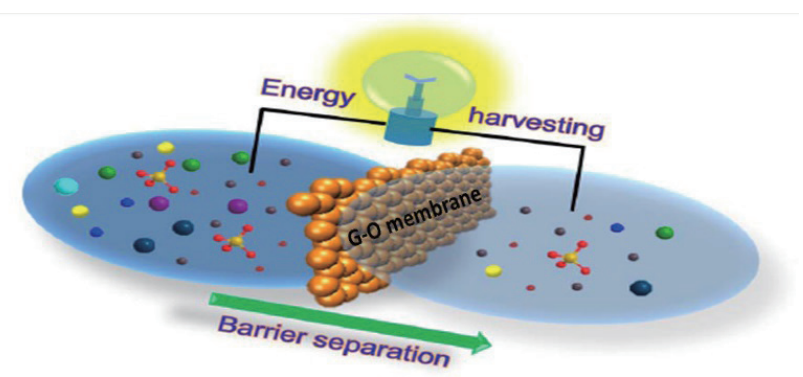

Fig. 8. (Color online) Transport of charges through bulk membranes. ${ }^{(61,62)}$ 
The value of $\lambda$ for specific species depends mainly on the chemical composition of the transport medium. This is attributed to the fact that the interactions between functional groups on the transport medium can alter the diffusivity, and hence the mobility, of the transported species. Accordingly, the values of $\lambda_{i}$ are often reported for pure electrolytes and extrapolated to infinite dilution. Moreover, some references ${ }^{(53,63)}$ use the ionic mobility of species $\mu_{i}\left(\mathrm{~cm}^{2} / \mathrm{Vs}\right)$ instead of $\lambda_{i}$, which is related to $\lambda_{i}$ by

$$
\lambda_{i}=z_{i} F_{i} \mu_{i}
$$

Actually, sometimes the following equation is used to replace the conventional NernstPlanck equation in the case of the transport of species through saturated porous structures in which the pores are mainly occupied with fluids, fluid cavities, and bubbles:

$$
\frac{\partial}{\partial t}\left(\theta C_{i}\right)+\frac{\partial}{\partial t}\left(\rho_{b} C_{P i}\right)+\frac{\partial}{\partial t}\left(a_{\nu} C_{G i}\right)=R_{i}+S_{i}+\nabla \cdot\left[\left(D_{D, i}+D_{e, i}\right) \nabla c_{i}\right]
$$

In this equation, $\theta$ stands for the liquid volume fraction, $C_{i}$ is the concentration of the $i$ th species per unit of fluid volume, $\rho_{b}$ is the bulk density calculated as $(1-\varepsilon) \rho_{p}, C_{p}$ is the concentration of the $i$ th species per unit mass of the solid, $a_{v}$ is the resulting gas volume fraction and is calculated as $\varepsilon-\theta$, and $C_{G}$ is the concentration of the $i$ th species per unit of gas volume.

\section{Multilayer and Structured Pore Clusters as Transport Medium}

In many cases, the architecture of bioelectronic devices involves a multilayer structure and/ or structured pore clusters, such as multilayer graphene cross-linked with graphene oxide used for water desalination [Fig. 9(a)]. In this illustration, the membrane is based on a graphene

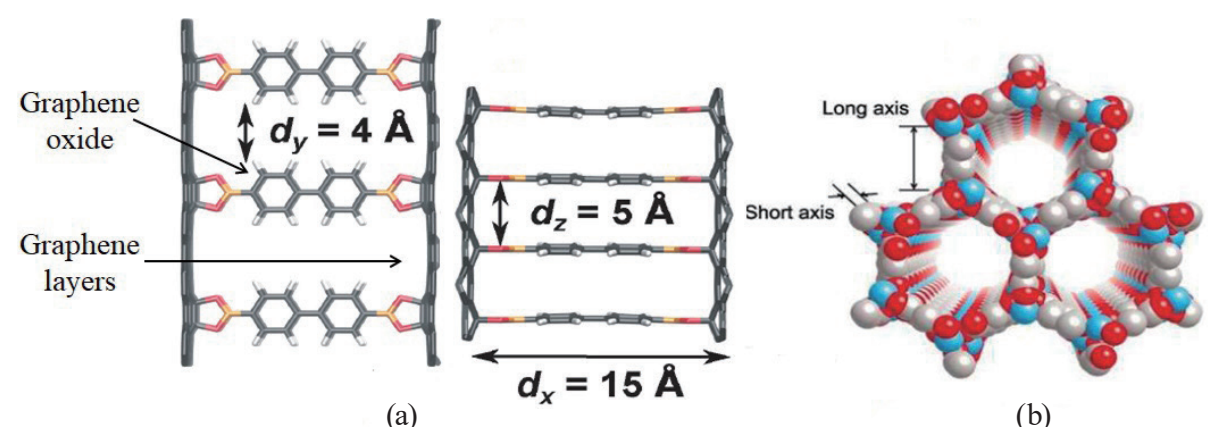

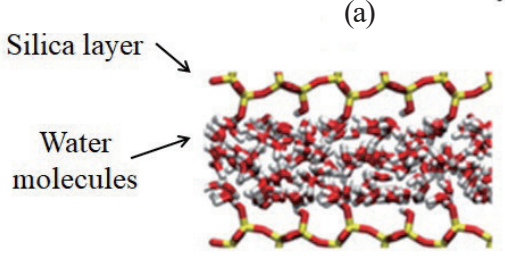

(c)

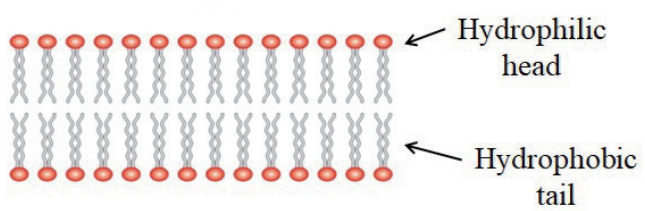

(d)

Fig. 9. (Color online) (a) Multilayer graphene cross-linked with graphene oxide used for water desalination, ${ }^{(64)}$ (b) structured pore clusters of graphenelike organic metal membrane for drug delivery, ${ }^{(1)}$ (c) binary silica layer for water transport, ${ }^{(66)}$ and (d) lipid bilayer. ${ }^{(62)}$ 
oxide framework (GOF) set perpendicular to the graphene layers. In the case of the bulk, GOF membranes have a finite length along the graphene layers. The entire structure appears to be intervallic along the $x$ - and $y$-axes. The transport of particles through this type of GOF membrane depends on the concentration of cross-linkers, where the number of cross-linker strands is in the range of 16 to 64 . This dependence was attributed to the fact that the distances $d_{x}$ and $d_{y}$ between successive cross-linkers in the $x y$ plane (considered here as the pore size) and the distance $d_{z}$ between successive cross-linkers in the $x z$ plane (pore spacing) determine the free volume of transport. The higher the number of cross-linkers, the smaller the pore size. Taking into consideration that $d_{x}$ is fixed (based on the type of cross-linker), the free volume for transport is reduced in the case of a high concentration of cross-linker. ${ }^{(64)}$ An example of a structured pore transport medium is the structured pore clusters of graphenelike organic metal membranes for drug delivery [Fig. 9(b)]. As shown in Fig. 9(b), a metal-organic framework (MOF-74) composed by 2,5-dihydroxyterephthalate and $\mathrm{Mg}(\mathrm{II})$ ion is used to deliver two anticancer drug molecules, methotrexate (MTX) and 5-fluorouracil (5-FU). ${ }^{(65)}$ Such a MOF structure has the ability to have its transport properties controlled by adapting the organic linker or metal ion while maintaining its main architecture (similar to the GOF-based membranes). In fact, in most MOF architectures, the length of the organic linker is considered a design parameter; however, this parameter does not allow much control because the length of the linker depends on the chain type. The main ability to modify is attributed to changing the metal ion, as changing the metal strongly affects the coordination geometry and consequently the transport properties. ${ }^{(11)}$ Other examples of multilayered and pore-structured transport media are binary silica layers for water transport [Fig. 9(c)] ${ }^{(66)}$ and transport though binary biolayers of functional groups such as lipid bilayers ${ }^{(62)}$ [Fig. 9(d)], which may be used in the design of bioelectronic devices.

In this case, the Nernst-Planck equation cannot solely describe the transport of species through the structures. It only enables the implementation of a single scalar electric field computation as it interrelates only the charge, ionic mobility, and concentration of the species along the boundaries of the transport medium. The Poisson-Nernst-Planck (PNP) theory, as a continuum-based coupled model that describes the dynamics of ions and the evolution of the electric field inside an electrolyte, may be used as an alternative. The PNP equations are derived from the conventional Nernst-Planck transport equations coupled with Poisson's field equation. By this method, it is possible to describe an electrolyte solution via two scalar fields by considering a binary transport medium that can dissociate water into cations and anions that fill the entire transport medium. In this scenario, the PNP theory tends to reduce all electrostatic interactions between ions to Coulombic interactions between ions and a meanfield electric potential, $\psi$. This yields a very descriptive assumption that mimics the transport dynamics, and hence, the conservation of species concentration may be expressed as ${ }^{(53,55)}$

$$
\frac{\partial c_{i}}{\partial t}=-\nabla \cdot J_{i}
$$

Here, $J_{i}$ is the total flux of species. In such a case, $i$ may be either negative or positive, and in the absence of fluid motion, $J_{i}$ is given by 


$$
J_{i}=-D_{i} \nabla c_{i}-b_{i} z_{i} e \nabla \psi c_{i}
$$

The right-hand side of Eq. (12) represents the previously mentioned diffusive flux, coupled with the electromigrative flux that takes into account the motion of ions as follows:

$$
\begin{gathered}
\text { Diffusive flux }\left(\phi_{\text {diff }, i}\right) \rightarrow-D_{i} \nabla c_{i}, \\
\text { Electro-migrative flux }\left(\phi_{\text {mig, }, i}\right) \rightarrow-b_{i} z_{i} e \nabla \psi c_{i} .
\end{gathered}
$$

It can be seen that the electromigrative flux term considers the transport of ions to be due to the electrostatic interactions between the electric field, $E=-\nabla \psi$, and the electric charge on the ion, $z_{i} e$, where $z_{i}$ is the ion's valence and $e$ is its fundamental charge. In addition, the mobility coefficient $b_{i}$ correlates the drift velocity of ions to the electric force exerted on it, which is attributed to the nature of the grad operator. This computational privilege is missed in the Nernst-Planck fundamental model. Finally, the mean-field potential $\psi$ is related to the ion concentration and the permittivity coefficient of the transport medium, $\varepsilon$, via Poisson's equation, as follows:

$$
\nabla \cdot(\varepsilon \nabla \psi)=-\sum_{i} z_{i} c_{i} e
$$

As can be seen in Eq. (15), applying the curl operator to the dot product of the gradient of the mean-field potential and the medium permittivity is practically a descriptive technique. It physically results in assigning normal and tangential field vectors to the cavity's boundaries as well as distributing the charges of the functional groups on the medium all over the cavities' walls. When this expression is combined with the right-hand side of Eq. (15), the dynamics of transport of ionic species based on their interaction with the transport medium can be accurately computed and described.

\section{Transport in Nanoporous Membranes, Composite Electrodes, and Nanochannels}

Transport in nanopores and channels takes place in many bioelectronic devices. Figure 10(a) shows an example of a nanopore introduced to a single layer of graphene for DNA sequencing. It shows a single-stranded DNA transported through a nanopore in a graphene monolayer, where the diameter of the nanopore is around $1.5 \mathrm{~nm}$, corresponding to about 35 carbon rings. The strand is vertically transported under the effect of an applied electric potential. DNA should be transported through the pore by the effect of ionic flow (vertical yellow shading), and hence the characteristic changes in the ionic current caused by each type of DNA base may be measured through the graphene nanopore. ${ }^{(67)}$ Figure 10(b) shows catalyst-decorated 3D graphene composite electrodes for a biofuel cell in the form of a nano-honeycomb-like strongly coupled $\mathrm{CoMoO}_{4}-3 \mathrm{D}$ graphene hybrid structure where graphene replicates the perfect 3D porous network of a compressed foam. The width of the graphene network is in the range 


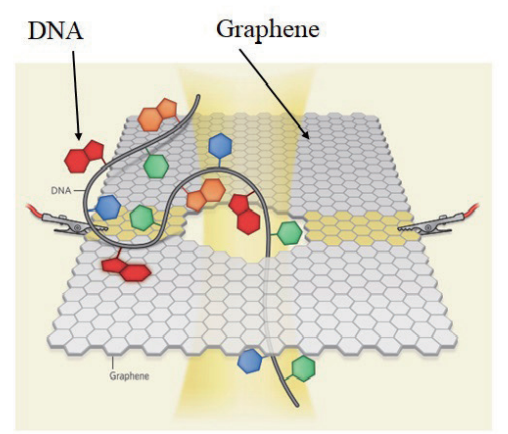

(a)

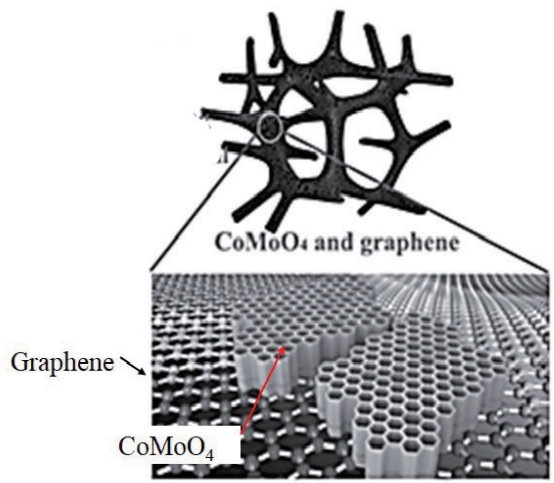

(b)

Fig. 10. (Color online) (a) A nanopore introduced to a single layer of graphene for DNA sequencing, ${ }^{(64)}$ and (b) catalyst-decorated 3D graphene composite electrodes for biofuel cells. ${ }^{(67,68)}$

of 100 to $120 \mu \mathrm{m}$. The graphene network is uniformly covered with the $\mathrm{CoMoO}_{4}{ }^{(68,69)}$ The transport modes and hence the dynamics are quite different compared with bulk transport. In this section, the principles of the transport of species in nanopores and nanochanneled media are introduced. A good practice to discuss these principles is to consider a nanochannel with positively charged inner walls, which bridges two domains of a given species with identical concentrations as shown in Fig. 11(a). When a charged surface is immersed into an electrolytic medium, anions are attracted to its walls and accumulate in its inner walls forming an electrical bilayer. ${ }^{(70,71)}$ Nanopored graphene located in between the charged inner walls can act as the selective membrane of the translocating ions. In order to mimic chemical selectivity by the biological channels, the size of the synthetic pores must be measurable with the diameter of the diffusing ions; hence, nanopored graphene is the best candidate owing to the minimal thickness of graphene. ${ }^{(70)}$ A critical parameter for transport through nanochannels is channel width because the diameter of the channel dictates the thickness of the double layer. In fact, it is the DL of the solution that dictates the thickness of the electrical bilayer, not the diameter of the transport channel. The DL is the distance over which the electrostatic coupling between the charged species in the solution takes place because of the identical concentration of the charges. Consequently, the transport channel in Fig. 11(a) has a radius that is much larger than the DL, and the center of the channel is occupied by both types of charges at the same concentration, as shown in Fig. 11(b), while the peripheral negative charges accumulate at the channel walls. If the radius of the channel is short compared with the DL, then the concentration of negative ions dominates the channel's cross section as depicted in Fig. 11(c). ${ }^{(70)}$

In such an environment, where changes in species velocity are very sensitive to any change exerted by the forces of the medium, the transfer of momentum between ions and solvent is better explained by the Navier-Stokes equation:

$$
\rho\left(\frac{\partial v}{\partial t}+v \cdot \nabla v\right)=-\nabla P+\eta \nabla^{2} v+f .
$$




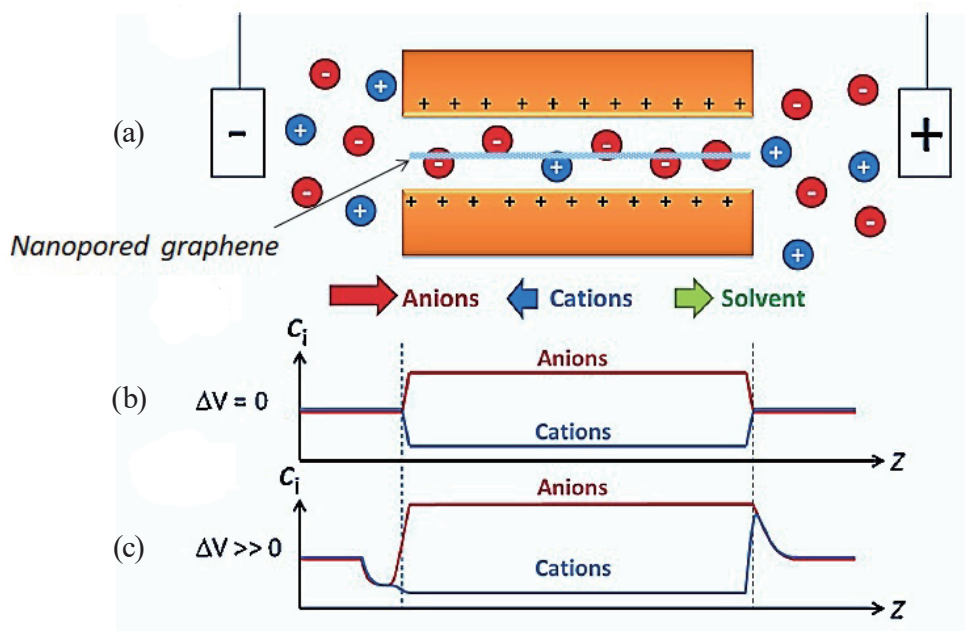

Fig. 11. (Color online) (a) A transport nanochannel with positively charged inner walls, (b) charge concentration profiles in a nanochannel with a radius much larger than the Debye length, and (c) charge concentration profiles in a nanochannel with a radius less than twice the Debye length. ${ }^{(70)}$

Here, the velocity of the diluted species, $v$, and the species density $\rho$ are related to the pressure inside the channel, $p$, the viscosity of the transport medium, $\eta$, and the volume force acting on the fluid, $f$, in one governing equation. As can be observed from Eq. (16), three components represent the normal forces: the viscosity embeds the tangential sheer forces in the model, and the volume force that may contain gravitational, electrostatic, and magnetic forces. However, in the current scenario, $f$ is the force that the electric field exerts on the ions in the fluid, namely, $f=-\rho_{Q} \nabla \psi$, where $\rho_{Q}$ is the total charge density.

In the Navier-Stokes equation given by Eq. (16), because the pressure appears only as a gradient, the absolute magnitude of the pressure has no effect on the transport; in fact, it is only the pressure differences along the DL of the pore that affect the transport. Moreover, because in bioelectronic applications, the fluids are mostly incompressible, the force acting on the fluids in the absence of an electric field can be represented as

$$
f=\rho g,
$$

where $g$ is the acceleration due to gravity.

The set of PNP equations, coupled with the Navier-Stokes equation under the appropriate boundary conditions, has been widely used to model ion transport for many examples of solidstate nanochannels and nanopores. ${ }^{(63,72)}$ Recent computational studies coupling both models have enabled the description of complicated systems with very low free-volume structures such as grafted polyelectrolytes. ${ }^{(63,72)}$ This set of equations, owing to its description of the ion fluxes, as a continuum, is appropriate for cases when the radius of the channel is larger than double the DL. ${ }^{(73)}$ However, modelling pores and channels with radii less than twice the DLs (which is the case in biological ion channels) requires particle-based methods, such as molecular 
or Langevine dynamics or quantum chemistry calculations. In fact, coupling the Navier-Stokes equation with the PNP equations led to an accurate prediction of the transport properties of different species among porous structures.

For instance, Fig. 12 shows the calculated and measured $I-V$ curves for graphene membranes where lithium chloride, caesium chloride, and potassium chloride transported through nanopores of the membrane. These calculated $I-V$ curves presented in Fig. 12 resulted from a published simulation carried out using Matlab where the domain boundary was assumed to consist of an inlet, an outlet, and an impermeable lateral boundary. At the inlet boundary, velocity and concentrations were specified. The outlet boundary was subjected to constant pressure and a free exit condition for component concentrations. The lateral boundary was impermeable to flow and may not have been reactive. The domain consists of solid grains and pore space occupied entirely by a single liquid phase. The system of equations is discretized in the pore space of the domain using a conservative finite volume method on a Cartesian grid.

As can be seen in Fig. 12, the results calculated using the Navier-Stokes equation with the PNP equations align perfectly with the measured values for linear and quasi-linear transport performance. However, as the actual transport performance tends to be nonlinear, for negative transmembrane potentials, the calculations diverge. This divergence is attributed to the approximations used to solve the continuum set of equations numerically. Furthermore, the only constant in the computation algorithm that represents the membrane is the diffusivity. The diffusion coefficient is usually calculated on the basis of the famous Einstein formula, and $3 \mathrm{D}$ dissimilarity is neglected during calculations. In other words, the Einstein diffusivity calculations assume that the diffusion is similar in all directions in one plane. Figure 13(a) shows an interface layer of zinc oxide $(\mathrm{ZnO})$ nanorods on graphene. Such a combination was reported as a glucose biosensor. ${ }^{(74,75)}$ The $\mathrm{ZnO}$ branch is not symmetric, which resulted in an asymmetric electrostatic field around the atoms. Consequently, the diffusion of the glucose into the interface was direction dependent.

Furthermore, the electrostatic potentials and mean fields shown in Fig. 13(a), calculated using DFT, show that even the electrostatic properties for such sensor are isotropic, which is not the case for the Poisson calculated field. Various attempts have been made recently to couple the

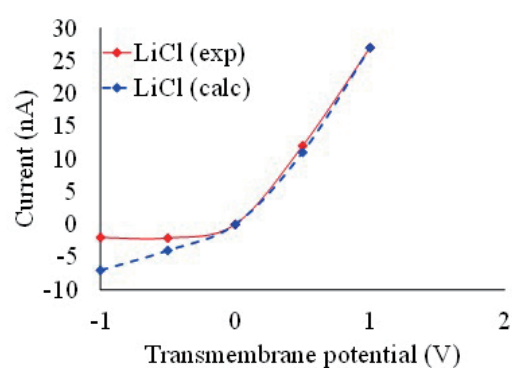

(a)

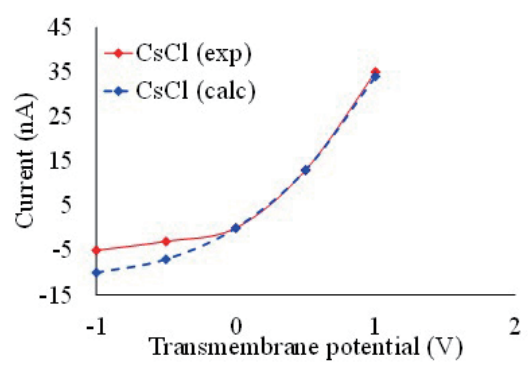

(b)

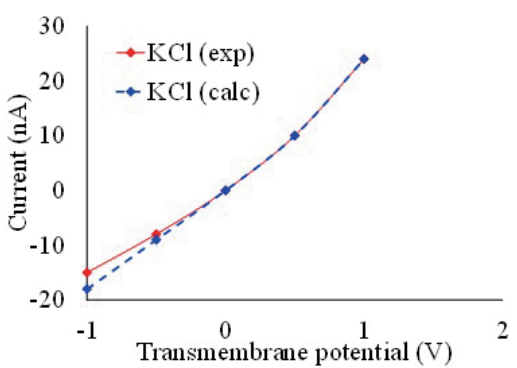

(c)

Fig. 12. (Color online) Calculated and measured $I-V$ curves for graphene membranes when (a) lithium chloride, (b) caesium chloride, and (c) potassium chloride were allowed to transport through nanopores of graphene membrane. ${ }^{(70)}$ 


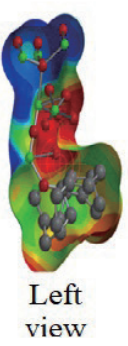

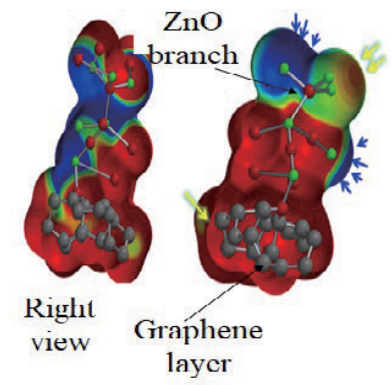

(a)

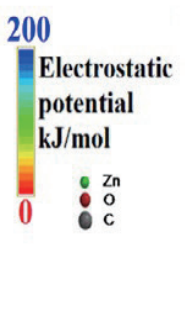

Electrostatic $\mathrm{kJ} / \mathrm{mol}$

$\mathrm{Zn}$
0
$\mathrm{c}$

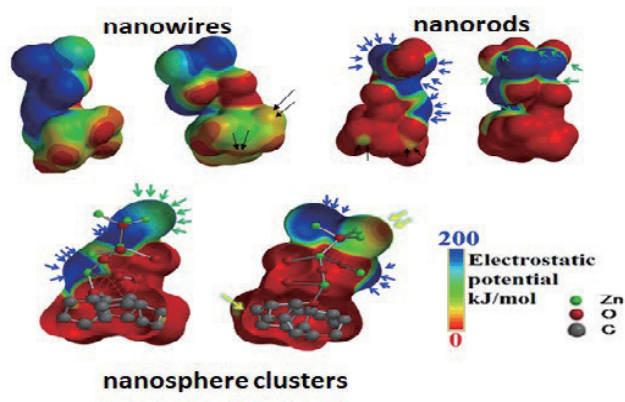

(b)

Fig. 13. (Color online) (a) Electrostatic potential maps for $\mathrm{ZnO}$ nanostructures grown on single-layer graphene, and (b) electrostatic potential map for $\mathrm{ZnO}$-decorated graphene sensor based on $\mathrm{ZnO}$ nanowires, nanorods, and nanosphere clusters using density functional theory. ${ }^{(75)}$

Nernst-Planck equations with DFT calculations, rather than with the Poisson model. Moreover, the continuum PNP equations that predict the macroscale properties of transport based on the microscopic properties of devices neglect the volumetric changes in the structure. Figure 13(b) shows the structures and electrostatic potential maps for a $\mathrm{ZnO}$-decorated graphene biosensor based on $\mathrm{ZnO}$ nanowires, nanorods, and nanosphere clusters. As can be seen in the figure, the electrostatic potential depends strongly on structure, as has been mentioned in our work. ${ }^{(75-77)}$ We found that the transport time for electrons differs by about 10 orders of magnitude between nanorods and nanosphere clusters. ${ }^{(75)}$

The use of graphene as an interface layer in the $\mathrm{ZnO}$-decorated sensor is the main reason behind the sensitivity of the transport properties to the volumetric changes in the structures. Detailed information based on X-ray absorption measurements (XAS), from the bulk and the surface on the degree of carbon $\mathrm{sp}^{3} / \mathrm{sp}^{2}$ hybridization and oxygen functional groups in two different $\mathrm{C}$ thin films is presented in the following. Although the surfaces of the two films are identical with respect to the $\mathrm{sp}^{3}$ to $\mathrm{sp}^{2}$ ratio, the differences in the $\mathrm{sp}^{2}$ content in the bulk make the $\mathrm{C}$ thin films electrically dissimilar. Such phenomena make graphene a promising material for biosensing. ${ }^{(78)}$ Other examples of DFT studies have described the detection of dopamine $(\mathrm{DA}),{ }^{(74)}$ uric acid (UA), ${ }^{(74)}$ and ascorbic acid (AA) ${ }^{(74)}$ levels in blood using a AgO-G biosensor. In those studies, the interactive forces between Ag nanoparticles and analytes (DA, UA, and AA) were investigated through a molecular orbital study where the positions of the highest occupied molecular orbital (HOMO) and lowest unoccupied molecular orbital (LUMO) in the system were determined. Consequently, the band gap (the difference between the HOMO and the LUMO) and the affinity patterns as well could be identified. In those studies, DFT calculations were performed at the B3LYP/LANL2DZ level of theory using the Gaussian 09 package to investigate the optical changes caused by the interaction of the Ag nanoparticles with UA, AA, and DA. ${ }^{(74)}$ To perform these calculations, the optimized geometries of the DA, $\mathrm{UA}$, and AA molecules were attached to a nanoarray of Ag atoms representing the substrate. Accordingly, B3LYP/LANL2DZ functional and basis sets were employed on the molecular model, and the absorption energies were determined by time-dependent DFT (TD-DFT) calculations. The results are illustrated in Fig. 14, where the lowest energy of the optimized 


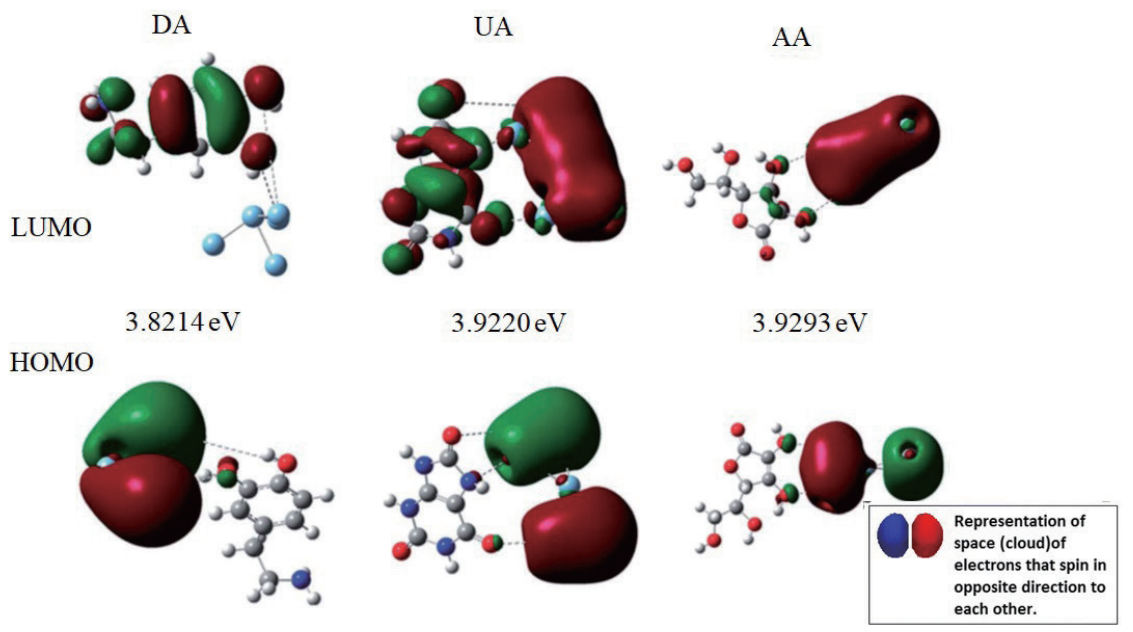

Fig. 14. (Color online) Calculated LUMO and HOMO energy states for Ag nanoparticles interacting with UA, AA, and DA using DFT-B3LYP/LANL2DZ level of theory. ${ }^{(75)}$

geometries of the $\mathrm{Ag}$ nanoparticles with DA, UA, and AA are presented. It can be seen that the LUMO is positioned near the dopamine side in the Ag-DA case, while in the case of the AgUA adduct, the LUMO is positioned at both sides of the Ag and UA branches. In contrast, the LUMO is located at the Ag side with a slight inclination towards the AA group in the case of the Ag-AA adduct. Finally, the HOMO is mainly located on the Ag side. ${ }^{(74)}$

The HOMO-LUMO energy separation has been used as a simple indicator of kinetic stability and may indicate the affinity pattern of the molecule. A reasonable HOMO-LUMO energy gap (3.8214 eV for Ag-DA, $3.9220 \mathrm{eV}$ for $\mathrm{Ag}-\mathrm{UA}$, and $3.9293 \mathrm{eV}$ for $\mathrm{Ag}-\mathrm{AA}$ ), showing a reasonably high affinity for DA with Ag and a lower affinity for UA and AA, agrees with the fact that it is energetically unfavourable to add electrons to a high-lying LUMO or to extract electrons from a low-lying LUMO. The simulated spectra of the Ag nanoparticles with DA, UA, and AA obtained from the TD-DFT calculations concur with the experimental absorption spectra. Hence, it is clear that the changes in the surface plasmon resonant (SPR) responses of Ag nanoparticle clusters with DA, UA, and AA due to the binding of these species lead to the formation of an internal charge transfer (ICT) complex. DFT studies gave further evidence for the higher detection limit of DA due to the higher binding with Ag nanoparticles compared with the others, as confirmed by the energy gap. A similar study was conducted for the detection of DA using a magnetite-graphene biosensor. ${ }^{(79)}$ It was found that a DA molecule could be strongly physisorbed on the $G$ surface in various orientations. The orientations of the DA molecule on the G surface may affect the active sites for interactions. The Stone-Wales defect on graphene could affect the interactions between the DA molecule with specific orientations and the graphene surface. Generally, the effect of defects on the interactions between DA and DG was not obvious. ${ }^{(79)}$ For the DA-GO systems, the DA molecule might be chemisorbed on the GO surface in specific orientations. For the DA-Fe-G systems, the interactions between the DA molecule and the Fe-G surface can be improved by doping the surface with a Fe atom bound to DA in their configuration. This indicates that graphene-based materials with specific 
structures or chemical groups may be designed to satisfy the demands for different applications of graphene-based materials. The interactions between DA and graphene could be adjusted by chemical or physical methods. This research is very meaningful for designing specific graphene-based materials for DA sensing and DA/graphene composites.

A similar study found that oxygen-functionalized structures in graphene oxide lead to a small band gap that made the graphene more sensitive to the materials in the environment so it could be placed on top of the Au layer in a typical surface plasmon resonance sensor to improve the sensitivity of that sensor. In this case, variations in carrier density affected the graphenebased SPR sensor response. In addition, in the presence of different organic molecules, the refractive index shift was determined and the molecular properties of each sensing material such as electronegativity, molecular mass, and effective group number were considered. On the basis of these parameter sets, the analysis was performed simultaneously and the related coefficients were reported. A semiempirical model for the interpretation of changes in the SPR curve has also been suggested and tested for some organic molecules. ${ }^{(80)}$

According to the results of determining the adsorption energy $E_{a d s}$, the interactions between a DA molecule and an Fe-G surface were improved when compared with those of the other three graphene systems, i.e., pristine, defected, and GO, as shown in Fig. 15. The data indicated that Fe could be helpful in strengthening the interactions between DA and graphene sheets. To further study the interactions between a DA molecule and the Fe-G surface, the electron density

(a)

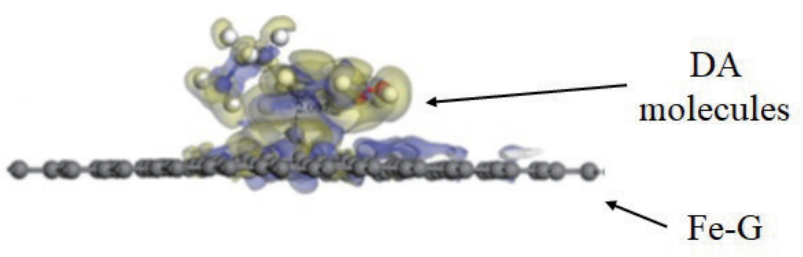

(b)

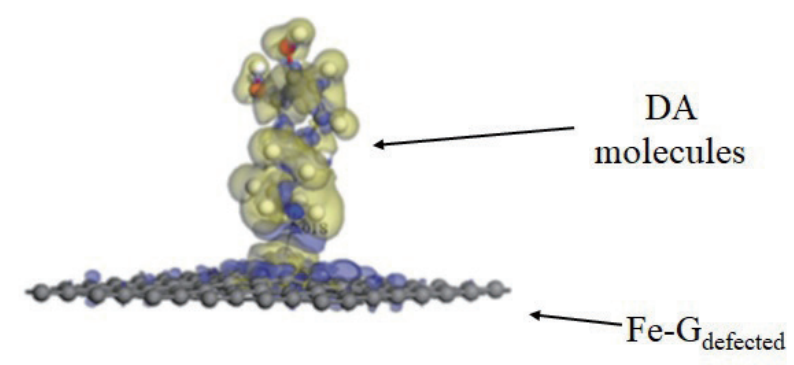

(c)

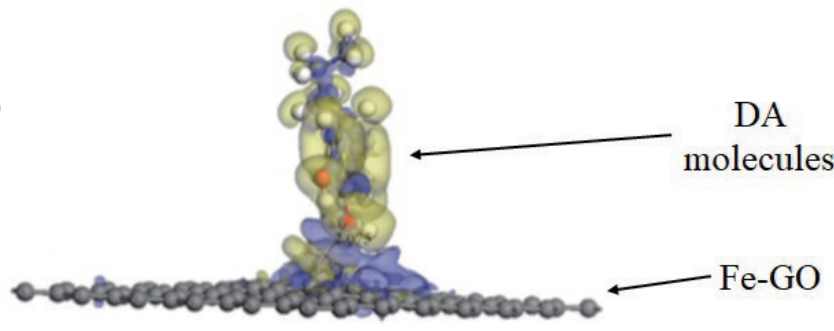

Fig. 15. (Color online) Interactions between a DA molecule and an Fe-G surface in cases of (a) pristine graphene, (b) graphene with defects, and (c) graphene oxide. ${ }^{(79)}$ 
difference was explored. An obvious electron transfer could be seen from Fe in the Fe-G sheet to the DA molecule. The number of electrons transferred was closely related to the interaction between the sheet and the molecule. When a DA molecule lay on the Fe-G surface, it had the largest interactions with the Fe-G surface and also exhibited the most apparent electron transfer (Fig. 15). Thus, the results of the $E_{a d s}$ determination corresponded to those of the electron density difference. ${ }^{(79)}$

\section{Conclusions}

In this short review, the main types of bioelectronic devices found in the literature were classified and investigated from the viewpoint of their design. The analysis of the architecture and design of graphene-based bioelectronic devices was considered and discussed using a computational analysis of the charge transport properties. The analysis was carried out on both the macroscale and nanoscale. The design of devices was investigated on the basis of approaches using FEA, DFT, and coupled multiphysics models. The impact of design on the performance of the bioelectronic device was also discussed. Moreover, a study of transport phenomena with respect to various structures of bioelectronic devices was conducted to clarify the charge transport mechanisms for several design categories. Finally, the published results of computed and measured charge transport properties of graphene-based bioelectronic devices were compared.

\section{Acknowledgments}

A.A.A. and N.N.N.M.I. thank the Universiti Teknologi Malaysia for providing a postdoctoral fellowship and scholarship, respectively. This work was funded by the Malaysia-Japan International Institute of Technology; Universiti Teknologi Malaysia; the Malaysian Ministry of Science, Technology and Innovation; and the Malaysian Ministry of Education through various research grants.

\section{Authors' Contributions}

A.A.A. and N.N.N.M.I provided the ideas and structure of the entire article and drafted the manuscript. AC completed the sections on "DFT studies describing reactions in SPR". AMH participated in the data analysis, discussion, and revision of the manuscript. All authors read and approved the final manuscript.

\section{Competing Interests}

The authors declare that they have no competing interests. 


\section{References}

1 S. Prabhulkar, H. Tian, X. Wang, J.-J. Zhu, and C.-Z. Li: Antioxid. Redox Signaling 17 (2012) 1796.

2 G. Slaughter and T. Kulkarni: J. Biochip Tissue Chip 5 (2015) 110.

3 S. A. Neto and A. R. De Andrade: J. Braz. Chem. Soc. 24 (2013) 1891.

4 M. Hussain, J. Wackerlig, and P. A. Lieberzeit: Biosensors 3 (2013) 89.

5 J. W.-F. Law, N.-S. A. Mutalib, K.-G. Chan, and L.-H. Lee: Front. Microbiol. 5 (2014) 770.

6 C. Zhu, G. Yang, H. Li, D. Du, and Y. Lin: Anal. Chem. 87 (2015) 230.

7 O. S. Kwon, H. S. Song, S. J. Park, S. H. Lee, J. H. An, J. W. Park, H. Yang, H. Yoon, J. Bae, T. H. Park, and J. Jang: Nano Lett. 15 (2015) 6559.

8 J. Vidic, M. Manzano, C.-M. Chang, and N. Jaffrezic-Renault: Vet. Res. 48 (2017) 11.

9 J. Wikswo, E. L. Curtis, Z. E. Eagleton, B. C. Evans, A. Kole, L. H. Hofmeister, and W. J. Matloff: Lab Chip 13 (2013) 3496.

10 B. Chen, H. Ma, L.-Y. Qin, F. Gao, K.-M. Chan, S.-W. Law, L. Qin, and W.-H. Liao: J. Orthop. Transl. 5 (2016) 26.

11 M. D. Allendorf and V. Stavila: Cryst. Eng. Comm. 17 (2015) 229.

12 D. Grieshaber, R. MacKenzie, J. Vörös, and E. Reimhult: Sensors 8 (2008) 1400.

13 B. Muthuraj, S. Mukherjee, S. R. Chowdhury, C. R. Patra, and P. K. Iyer: Biosens. Bioelectron. 89 (2017) 636.

14 D. O. Hare: Biosensors and Sensor Systems (Springer-Verlag, London, 2014).

15 M. Pohanka and P. Skadal: J. Appl. Biomed. 6 (2008) 57.

16 L. Clark and C. Lyons: Ann. N. Y. Acad. Sci. 148 (1962) 133.

17 R. Renneberg, D. Pfeiffer, F. Lisdat, G. Wilson, U. Wollenberger, F. Ligler, and A. P. Turner: Adv. Biochem. Eng. Biotechnol. 109 (2008) 1.

18 J. L. Barredo, Ed.: Microbial Enzymes Biotransformations (Humana Press, Totowa, New Jersey, 2005).

19 P. Mehrotra: J. Oral Biol. Craniofac. Res. 6 (2016) 153.

20 J. Filip and J. Tkac: Electrochim. Acta. 136 (2014) 340.

21 N. J. Ronkainen, H. B. Halsall, and W. R. Heineman: Chem. Soc. Rev. 39 (2010) 1747.

22 Y. Liu, X. Dong, and P. Chen: Chem. Soc. Rev. 41 (2012) 2283.

23 H. S. Song, O. S. Kwon, J.-H. Kim, J. Conde, and N. Artzi: Biosens. Bioelectron. 89 (2017) 187.

24 H. H. Nguyen, J. Park, S. Kang, and M. Kim: Sensors 15 (2015) 10481.

25 M. K. Dubey, A. Zehra, M. Aamir, M. Meena, L. Ahirwal, S. Singh, S. Shukla, R. S. Upadhyay, R. BuenoMarí, and V. K. Bajpai: Front. Microbiol. 8 (2017) 1032.

26 K. S. Novoselov, D. Jiang, F. Schedin, T. J. Booth, V. V. Khotkevich, S. V. Morozov, and A. K. Geim: Proc. Natl. Acad. Sci. U.S.A. 102 (2005) 10451.

27 M. J. Allen, V. C. Tung, and R. B. Kaner: Chem. Rev. 110 (2010) 132.

28 V. Chabot, D. Higgins, A. Yu, X. Xiao, Z. Chen, and J. Zhang: Energy Environ. Sci. 7 (2014) 1564.

29 Z. Zhu, L. Garcia-Gancedo, A. J. Flewit, H. Xie, F. Moussy, and W. I. Milne: Sensors 12 (2012) 5996.

30 Z. Zhu: Nano-Micro Lett. 9 (2017) 25.

31 H. Pan and B. Chen: Sci. Rep. 4 (2014) 4198.

32 L. Wang, W. B. Ng, J. A. Jackman, and N.-J. Cho: Adv. Funct. Mater. 26 (2016) 2097.

33 R. Atif, I. Shyha, and F. Inam: Polymers 8 (2016) 281.

34 T. Musso, P. V Kumar, A. S. Foster, and J. C. Grossman: ACS Nano. 8 (2014) 11432.

35 W. Xin-ran, S. Yi, and Z. Rong: Chin. Phys. B. 22 (2013) 098505.

36 W. S. Lim, Y. Y. Kim, H. Kim, S. Jang, N. Kwon, B. J. Park, J.-H. Ahn, I. Chung, B. H. Hong, and G. Y. Yeom: Carbon 50 (2012) 429.

37 F. Yu, C. Wang, and J. Ma: Materials 9 (2016) 807.

38 M. Li, F. Pan, E. S. G. Choo, Y. Lv, Y. Chen, and J. Xue: ACS Appl. Mater. Interfaces 8 (2016) 6972.

39 T. Kulkarni and G. Slaughter: Membranes 6 (2016) 55.

40 S. Liu and X. Guo: NPG Asia Mater. 4 (2012) e23.

41 S. Mao, K. Yu, G. Lu, and J. Chen: Nano Res. 4 (2011) 921.

42 Y. Ohno, K. Maehashi, and K. Matsumoto: J. Am. Chem. Soc. 132 (2010) 18012.

43 A. C. M. de Moraes and L. T. Kubota: Chemosensors 4 (2016) 20.

44 H. Chen, J. Huang, A. Palaniappan, Y. Wang, B. Liedberg, M. Platt, and A. I. Y. Tok: Analyst 141 (2016) 2335.

45 C. S. Park, H. Yoon, and O. S. Kwon: J. Ind. Eng. Chem. 38 (2016) 13.

46 D. Huh, G. A. Hamilton, and D. E. Ingber: Trends Cell Biol. 21 (2011) 745.

47 E. W. Esch, A. Bahinski, and D. Huh: Nat. Rev. Drug Discov. 14 (2015) 248.

48 P. Bajaj, J. F. Harris, J.-H. Huang, P. Nath, and R. Iyer: ACS Biomater. Sci. Eng. 2 (2016) 473. 
49 M. Tehranirokh, A. Z. Kouzani, P. S. Francis, and J. R. Kanwar: Biomicrofluidics 7 (2013) 51502.

50 D. O. Freytes, L. Q. Wan, and G. Vunjak-Novakovic: J. Cell Biochem. 108 (2009) 1047.

51 N. Inoue and H. Onoe: J. Micromech. Microeng. 28 (2017) 014001.

52 Y. Song, C. Chen, and C. Wang: Nanoscale 7 (2015) 7084.

53 M. Mirzadeh and F. Gibou: J. Comput. Phys. 274 (2014) 633.

54 J.-O. Lee, H.-M. So, E.-K. Jeon, H. Chang, K. Won, and Y. H. Kim: Anal. Bioanal. Chem. 390 (2008) 1023.

55 B. Lu, M. J. Holst, J. A. McCammon, and Y. C. Zhou: J. Comput. Phys. 229 (2010) 6979.

56 T. Geyer: BMC Biophys. 4 (2011) 7.

57 M. O. Steinhauser and S. Hiermaier: Int. J. Mol. Sci. 10 (2009) 5135.

58 T. Yamaguchi, T. Ishikawa, Y. Imai, N. Matsuki, M. Xenos, Y. Deng, and D. Bluestein: Ann. Biomed. Eng. 38 (2010) 1225.

59 C. Picioreanu, J.-U. Kreft, and M. C. M. Van Loosdrecht: Appl. Environ. Microbiol. 70 (2004) 3024.

60 Z. Hemmatian, S. Keene, E. Josberger, T. Miyake, C. Arboleda, J. Soto-Rodriguez, F. Baneyx, and M. Rolandi: Nat. Commun. 7 (2016) 12981.

61 P. Sun, F. Zheng, M. Zhu, K. Wang, M. Zhong, D. Wu, and H. Zhu: Sci. Rep. 4 (2014) 5528.

62 G. J. Tortora, B. R. Funke, and C. L. Case: Microbiology: An Introduction (Pearson, San Francisco, 2014) 12th ed.

63 D. Barak-Shinar, M. Rosenfeld, and S. Abboud: J. Electrochem. Soc. 151 (2004) 261.

64 A. Nicola1, B. G. Sumpter, and V. Meunier: Phys. Chem. Chem. Phys. 16 (2014) 8646.

65 M. Bernini, D. Fairen-Jimenez, M. Pasinetti, A. J. Ramirez-Pastor, and R. Q. Snurr: J. Mater. Chem. B 2 (2014) 766.

66 S. H. Krishnan and K. G. Ayappa: J. Phys. Chem. B. 109 (2005) 23237.

67 S. Garaj, W. Hubbard, A. Reina, J. Kong, D. Branton, and J. A. Golovchenko: Nature 467 (2010) 190.

68 Q. Shi, Y. Cha, Y. Song, J.-I. Lee, C. Zhu, X. Li, M.-K. Song, D. Du, and Y. Lin: Nanoscale 8 (2016) 15414.

69 X. Yu, B. Lu, and Z. Xu: Adv. Mater. 26 (2014) 1044.

70 M. Tagliazucchi and I. Szleifer: Mater. Today 18 (2015) 131.

71 S. Scheiner, P. Pivonka, and D. W. Smith: Comput. Geotech. 48 (2013) 283.

72 S. Molins, D. Trebotich, C. I. Steefel, and C. Shen: Water Resour. Res. 48 (2012) W03527.

73 W. Sparreboom, A. van den Berg, and J. C. T. Eijkel: New J. Phys. 12 (2010) 015004.

74 K. Z. Kamali, A. Pandikumar, G. Sivaraman, H. N. Lim, S. P. Wren, T. Sun, and N. M. Huang: RSC. Adv. 5 (2015) 17809.

75 A. A. Ali and A. M. Hashim: Nanoscale Res. Lett. 11 (2016) 246.

76 A. A. Ali and A. M. Hashim: Nanoscale Res. Lett. 10 (2015) 452.

77 A. A. Ali and A. M. Hashim: Nanoscale Res. Lett. 10 (2015) 299.

78 S. Sainio, D. Nordlund, M. A. Caro, R. Gandhiraman, J. Koehne, N. Wester, J. Koskinen, M. Meyyappan, and T. Laurila: J. Phys. Chem. C. 120 (2016) 8298.

79 H.-P. Zhang, X.-Y. Lin, X. Lu, Z. Wang, L. Fang, and Y. Tang: Mater. Chem. Front. 1 (2017) 1156.

80 B. Meshginqalam, H. Toloue, M. T. Ahmadi, A. Sabatyan, A. Centeno, and R. Ismail: Opt. Quantum Electron. 48 (2016) 328. 